\title{
Utilização de mantenedor de espaço tipo banda-alça na perda precoce de um dente decíduo: relato de caso clínico
}

\author{
Use of a band-loop space maintainer in the early loss of a primary tooth: clinical case report \\ Uso de un mantenedor de espacio de banda-loop en la pérdida temprana de un diente temporal:
} informe de un caso clínico

Recebido: 28/11/2021 | Revisado: 02/12/2021 | Aceito: 03/12/2021 | Publicado: 12/12/2021

\author{
Carina Printes Lobato \\ ORCID: https://orcid.org/0000-0002-4815-8451 \\ Centro Universitário Fametro, Brasil \\ E-mail: carinaprintes@gmail.com \\ Mariana Silveira Brasil \\ ORCID: https://orcid.org/0000-0003-0472-8376 \\ Centro Universitário Fametro, Brasil \\ E-mail: marianaa.sbrasil@gmail.com \\ Helen Batista Menezes Pereira \\ ORCID: https://orcid.org/0000-0001-9710-5316 \\ Centro Universitário Fametro, Brasil \\ E-mail: helen_pereiras@yahoo.com.br \\ Joyce Ferreira do Nascimento \\ ORCID: https://orcid.org/0000-0002-4093-6669 \\ Centro Universitário Fametro, Brasil \\ E-mail: njole8343@gmail.com \\ Bruna Elizabete Rocha Nery da Costa \\ ORCID: https://orcid.org/0000-0001-7853-7420 \\ Centro Universitário Fametro, Brasil \\ E-mail: nerybruna07@gmail.com \\ Gabriela de Figueiredo Meira \\ ORCID: https://orcid.org/0000-0002-8285-8769 \\ Universidades Federal de Santa Maria, Brasil \\ E-mail: gabrielameira1@ hotmail.com \\ Nayhane Cristine da Silva de Oliveira \\ ORCID: https://orcid.org/0000-0003-2056-5853 \\ Centro Universitário Fametro, Brasil \\ E-mail: nayhane.oliveira@fametro.edu.br
}

\begin{abstract}
Resumo
Objetivo: Utilização de mantenedor de espaço tipo banda-alça na perda precoce de um dente decíduo. Metodologia: Partindo da permissão da paciente por meio do Termo de Consentimento Livre e Esclarecido (TCLE) em partilhar sua imagem para devido fim, o presente estudo trata de um relato de caso clínico de cunho analítico descritivo (Pereira et al., 2018). Relato de Caso Clínico: Paciente L.S.S.R., 4 anos, gênero feminino, melanoderma, compareceu à clínica odontológica do Centro Universitário Fametro acompanhada dos pais. À anamnese, a responsável relatou como queixa principal a presença de dor no elemento 74. Na primeira consulta, foi constatado pela responsável que a paciente sofria de asma e anemia. Em relação a alimentação, a paciente tomava leite puro e mingau na mamadeira, escovava os dentes três vezes ao dia, porém, não tinha o hábito de usar o fio dental. Também foi mencionado que a paciente teve o hábito de sucção de chupeta até os três anos de idade. Em seguida, realizou-se profilaxia, exame clínico e radiografia periapical dos elementos 55 e 74. Considerações Finais: A reabilitação em perda unilateral do primeiro molar decíduo com o uso do mantenedor de espaço banda-alça apresenta eficácia em seus resultados, impedindo a mesialização do dente adjacente e preservando o espaço para a adequada erupção dos dentes sucessores permanentes.
\end{abstract}

Palavras-chave: Cárie; Dente; Mantenedor de espaço.

\begin{abstract}
Objective: Use of a band-loop space maintainer in the early loss of a primary tooth. Methodology: Based on the patient's permission through the Informed Consent Form (FICF) to share their image for the proper purpose, this study is a clinical case report of a descriptive analytical nature (Pereira et al., 2018). Clinical Case Report: Patient L.S.S.R., 4 years old, female, black, attended the dental clinic at Centro Universitário Fametro accompanied by her parents. During anamnesis, the guardian reported the presence of pain in element 74 as the main complaint. In the first
\end{abstract}


consultation, the guardian found that the patient was suffering from asthma and anemia. Regarding food, one patient drank pure and porridge from a bottle, brushed milk three times a day, however, he was not in the habit of flossing. It was also created that a patient had the habit of sucking a pacifier until the age of three. Then, prophylaxis, clinical examination and periapical radiography of elements 55 and 74 were performed. Final Considerations: Rehabilitation in unilateral loss of the deciduous first molar with the use of the band-loop space maintainer is effective in its results, preventing mesialization adjacent tooth and preserving space for proper eruption of permanent successor teeth.

Keywords: Caries; Tooth; Space maintainer.

\begin{abstract}
Resumen
Objetivo: Uso de un mantenedor de espacio de banda-loop en la pérdida temprana de un diente temporal. Metodología: Con base en el permiso del paciente a través del Formulario de Consentimiento Informado (FICF) para compartir su imagen con el propósito adecuado, este estudio es un reporte de caso clínico de naturaleza analítica descriptiva (Pereira et al., 2018). Reporte de Caso Clínico: Paciente LRSS, 4 años, mujer, negra, acudió a la clínica dental del Centro Universitário Fametro acompañada de sus padres. Durante la anamnesis, el tutor informó como principal queja la presencia de dolor en el elemento 74. En la primera consulta, el tutor constató que el paciente padecía asma y anemia. En cuanto a la alimentación, un paciente bebió pura y papilla de biberón, cepilló leche tres veces al día, sin embargo, no tenía la costumbre de usar hilo dental. También se creó que un paciente tenía la costumbre de chupar un chupete hasta los tres años. Posteriormente, se realizó profilaxis, exploración clínica y radiografía periapical de los elementos 55 y 74. Consideraciones finales: La rehabilitación en pérdida unilateral del primer molar deciduo con el uso del mantenedor de espacio band-loop es efectiva en sus resultados, evitando la mesialización del diente adyacente y preservando el espacio para la correcta erupción de los dientes sucesores permanentes.
\end{abstract}

Palabras clave: Caries; Diente; Mantenedor de espacio.

\title{
1. Introdução
}

Os dentes decíduos têm um papel extremamente importante no sistema estomatognático, pois auxiliam a criança na mastigação, na fala, bem como no ato de ocluir, no desenvolvimento dos músculos da face e servem como guia de erupção para os sucessores permanentes (Silva et al., 2020; Guimarães \& Oliveira, 2017).

Durante a fase da dentadura decídua, a perda dentária precoce geralmente está associada à cárie e traumas. Quando da perda por cárie, relaciona-se a razão desta perda a má higienização bucal e alta ingestão de açúcares presentes na dieta, podendo afetar tanto a região posterior quanto anterior (Nóbrega et al., 2018).

$\mathrm{Na}$ infância, um dos principais motivos que pode contribuir para o aparecimento de lesões de cárie em crianças de tenra idade é o conteúdo de mamadeiras, principalmente durante a mamada noturna. A adição de açúcares ao conteúdo destes dispositivos e a não higienização dental após as refeições, contribui para o aumento na incidência dessas lesões (De Araújo et al., 2018).

No caso das lesões dentárias traumáticas, há um alto índice em crianças mais novas relacionado principalmente à falta de coordenação motora, podendo desta forma afetar principalmente a região anterior (Nóbrega et al., 2018; Dos Reis et al., 2018).

A avaliação radiográfica é indispensável para diagnosticar se um dente decíduo foi perdido precocemente. A perda é considerada prematura quando o germe do sucessor permanente não atingiu o estágio 8 de Nolla, ou seja, a formação de $2 / 3$ da raiz. Perder um dente decíduo antes do tempo correto pode ocasionar migração dos dentes adjacentes, deglutição atípica, modificações na fala, extrusão do dente antagonista e, por fim, a diminuição do perímetro do arco e consequente apinhamento do mesmo (Silva et al., 2020; Amorim et al., 2019).

Nos casos onde o elemento decíduo é perdido antes de sua esfoliação natural, é necessário a preservação do espaço para seu sucessor permanente, e para isso se faz necessária a intervenção por meio de dispositivos denominados mantenedores de espaço (Pawar, 2019; Witti et al., 2018).

O mantenedor de espaço conserva a oclusão ideal, uma vez que preserva o espaço a ser ocupado pelo dente permanente, podendo ser classificado de acordo com o tipo, a saber, fixo e removível, e de acordo com a sua função, funcional 
e não funcional. O primeiro restabelece função e estética. Já o segundo não impede a extrusão dos dentes antagonistas nem reestabelece função e estética. No que tange ao tipo, no fixo ocorre a cimentação de uma banda ortodôntica para ser instalada nos dentes pilares. Por outro lado, o removível é confeccionado com uma placa de acrílico e preso através de grampos, e além disso, pode ser retirado pelo paciente para a alimentação e higienização (Janson et al., 2013).

O mantenedor de espaço mais indicado para perdas unitárias precoces de molares decíduos é o de banda-alça, sendo um mantenedor do tipo fixo e não funcional, e pode ser indicado tanto para dentes do arco superior como inferior (Lira et al., 2019).

O aparelho de banda-alça é considerado de fácil confecção, com curtas sessões clínicas, além de utilizar material de baixo custo, o que compõe uma gama de vantagem para sua utilização em clínicas escola. Outra vantagem para a utilização deste dispositivo está na possibilidade da erupção do dente permanente, sem que ocorra a remoção do aparelho (Menegaz et al., 2015).

O banda-alça é frequentemente utilizado onde não há risco de extrusão do antagonista, por isso se faz necessário um bom diagnóstico para a escolha adequada do tipo de mantenedor de espaço ideal para cada paciente (Vinothini et al., 2019).

Sendo assim, a partir dos procedimentos realizados na clínica odontológica de um centro universitário de Manaus (Amazonas, Brasil), o objetivo deste trabalho é relatar um caso clínico acerca da perda precoce de um dente decíduo, e o uso de mantenedor de espaço do tipo banda-alça, para fins de preservação do espaço biológico visando a correta erupção do sucessor permanente e consequente prevenção de más oclusões.

\section{Metodologia}

Partindo da permissão da paciente por meio do Termo de Consentimento Livre e Esclarecido (TCLE) em partilhar sua imagem para devido fim, o presente estudo trata de um relato de caso clínico de cunho analítico descritivo (Pereira et al., 2018), realizado na clínica odontológica do CEUNI Fametro, na disciplina de estágio supervisionado em Clínica Integrada II, do curso de graduação do Centro Universitário Fametro.

\section{Resultados e Discussão}

Paciente L.S.S.R., 4 anos, gênero feminino, melanoderma, compareceu à clínica odontológica do Centro Universitário Fametro acompanhada dos pais. À anamnese, a responsável relatou como queixa principal a presença de dor no elemento 74 .

$\mathrm{Na}$ primeira consulta, foi constatado pela responsável que a paciente sofria de asma e anemia. Em relação a alimentação, a paciente tomava leite puro e mingau na mamadeira, escovava os dentes três vezes ao dia, porém, não tinha o hábito de usar o fio dental. Também foi mencionado que a paciente teve o hábito de sucção de chupeta até os três anos de idade. Em seguida, realizou-se profilaxia, exame clínico e radiografia periapical dos elementos 55 e 74.

No exame extra-oral, é possível observar que a paciente possui assimetria facial com desvio mandibular para o lado direito; é braquifacial, padrão II, perfil convexo e com selamento labial (Figura 1). 
Figura 1 - Fotografias iniciais da face (frontal, sorrindo e perfil).


Fonte: Arquivo pessoal (2021).

Durante o exame clínico intra-oral, observou-se que a paciente se encontrava em dentadura decídua com espaços primatas nos arcos superior e inferior, lesões cariosas cavitadas nos elementos 55 e 74 , fluorose nos dentes anteriores, hipomineralização de esmalte nos molares, relação de molares e caninos em classe II, e sobremordida (Figura 2).

Figura 2 - A) Fotografia inicial intraoral frontal; B) Fotografia inicial intraoral lateral direita; C) Fotografia inicial intraoral lateral esquerda; D) Fotografia inicial intraoraloclusal superior; E) Fotografia inicial intraoral inferior.

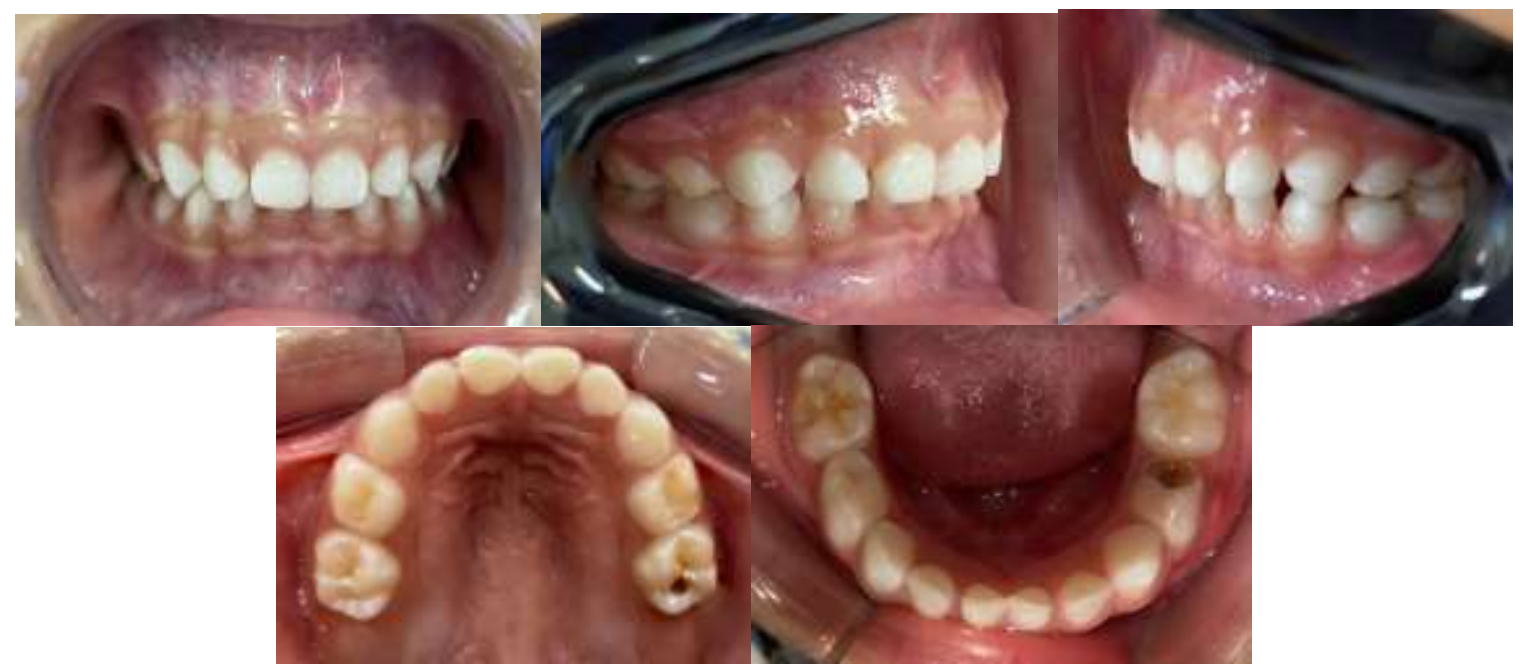

Fonte: Arquivo pessoal (2021).

No exame radiográfico periapical do elemento 74 , verificou-se lesão de cárie profunda e reabsorção óssea, sendo o mais indicado a exodontia. O elemento 34 encontrava-se no estágio 4 de Nolla (Figura 3).

Figura 3 - Radiografia periapical inicial.

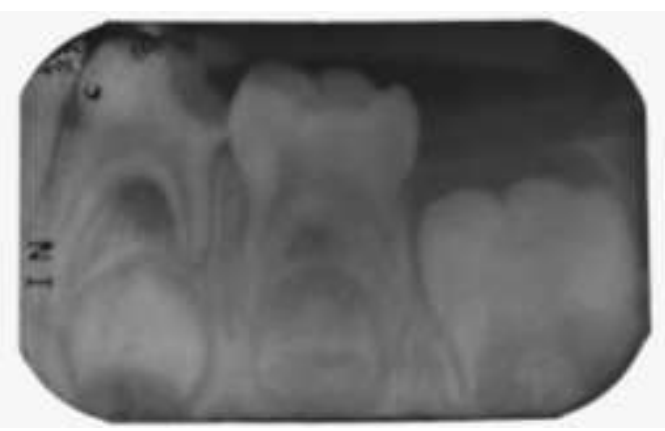

Fonte: Arquivo pessoal (2021). 
Em consultas posteriores, realizou-se o procedimento cirúrgico do elemento 74 utilizando-se lidocaína $2 \%$ com epinefrina 1:100.000 (Alphacaine 100), com número de tubetes calculados a partir do peso corporal da criança. No pósoperatório, foi prescrito paracetamol em gotas. As demais necessidades clínicas foram enquadradas em sessões posteriores, tal como a realização do procedimento restaurador no elemento 55 .

Logo após a cicatrização do alvéolo, efetuou-se a moldagem da arcada inferior com a banda ortodôntica número 32 posicionada no dente com auxílio do calcador de bandas. Utilizou-se moldeira infantil número 1 e alginato tipo II (Jeltrate Dustless). O anel ortodôntico foi estabilizado no molde através de fio ortodôntico para evitar erros durante o vazamento do gesso. Na sequência, o gesso pedra tipo III foi vazado no molde para a confecção do mantenedor de espaço tipo banda-alça com coroa de estoque (Figura 4).

Figura 4 - A) Moldagem com banda ortodôntica número 32; B) Mantenedor de espaço banda-alça com coroa de estoque.

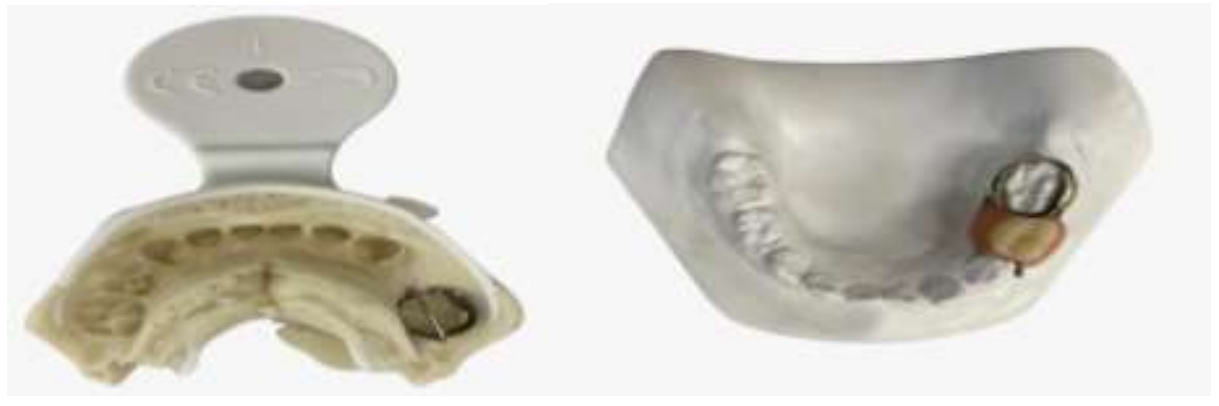

Fonte: Arquivo pessoal (2021).

A documentação ortodôntica foi solicitada e partir da análise da radiografia panorâmica, foi verificado que todos os elementos permanentes se encontravam em formação, e a paciente apresentava boa estrutura óssea da maxila e mandíbula os resultados obtidos na telerradiografia e análise cefalométrica USP, foram: classe II esquelética com protusão maxilar (Figura $5)$.

Figura 5 - A) Radiografia panorâmica; B) Telerradiografia USP; C) Traçado Cefalométrico USP.
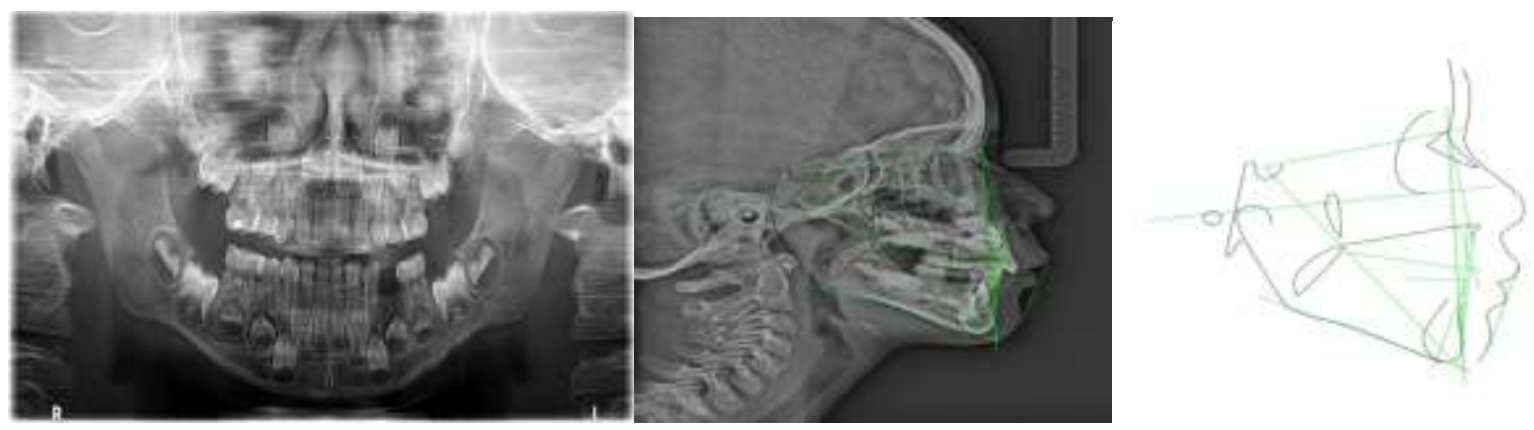

Fonte: CIMO.

Quando o aparelho foi adaptado ao dente da paciente, verificou-se que a coroa protética estava alta; sugeriu-se então um banda-alça convencional (Figura 6). Todo o processo de moldagem foi feito novamente para a confecção do mesmo. 
Figura 6 - Mantenedor de espaço tipo banda-alça convencional.

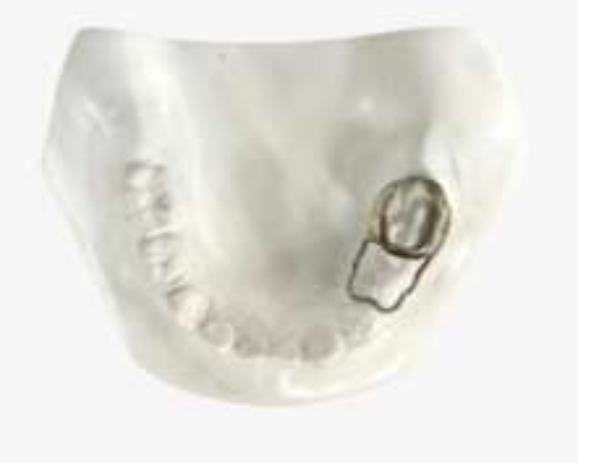

Fonte: Arquivo pessoal (2021).

Para a instalação do dispositivo, realizou-se nova profilaxia e o aparelho mantenedor de espaço banda-alça convencional foi cimentado no elemento 75 utilizando-se cimento de ionômero de vidro autopolimerizável (Maxxion $R$ ) (Figuras 7 e 8) e a paciente permaneceu em estado de proservação até o retorno no ano seguinte, em 2021, após 5 meses, na clínica de estágio supervisionado, para fazer o acompanhamento clínico e radiográfico.

Figura 7 - Cimento de ionômero de vidro autopolimerizável.

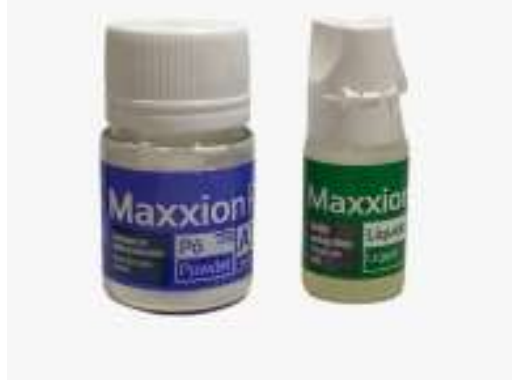

Fonte: Arquivo pessoal (2021).

Figura 8 - A: Fotografia mantenedor de espaço banda-alça oclusal; B: Fotografia mantenedor de espaço banda-alça lateral.

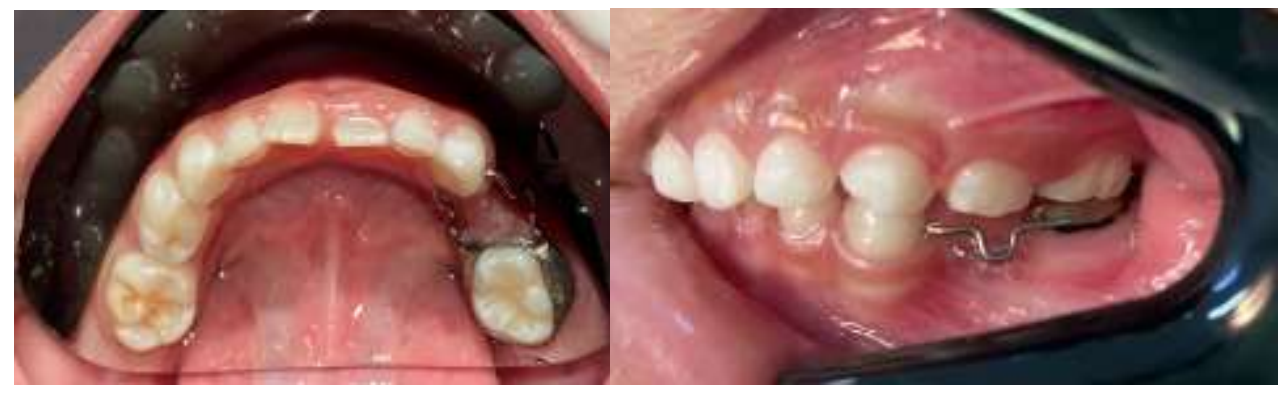

Fonte: Arquivo pessoal (2021).

Nesta consulta de retorno, não foram observadas alterações significativas como extrusão do dente antagonista e mesialização do elemento distal, portanto, a paciente deverá continuar em acompanhamento até que o dente permanente erupcione e só então ocorrerá a remoção do mantenedor de espaço. Somente após a remoção deste dispositivo, a paciente será submetida à avaliação ortodôntica para verificar a necessidade de outros tratamentos. 
Figura 9 - Radiografia periapical de acompanhamento.

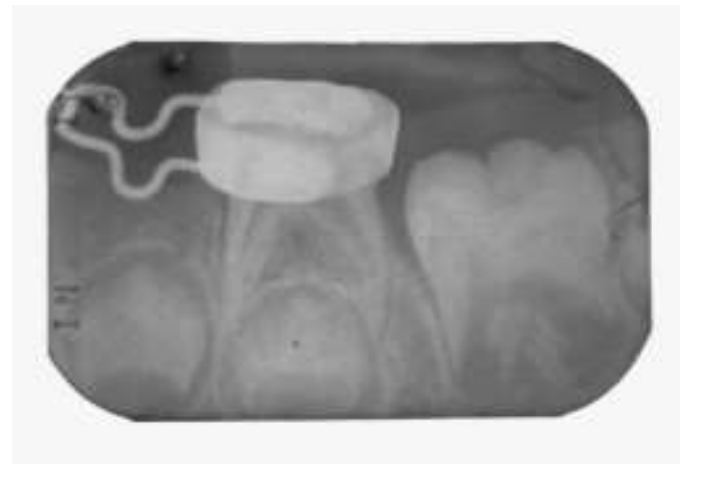

Fonte: Arquivo pessoal (2021).

\section{Discussão}

A perda prematura de dentes decíduos, antes da esfoliação fisiológica, poderá desencadear um desiquilíbrio oclusal, tendo como consequências: uma função mastigatória incorreta e diminuição da dimensão vertical no espaço edêntulo. Deste modo, o descuido com a dentição decídua poderá resultar em danos nocivos à qualidade de vida das crianças (EchevarríaMedieta et al., 2019; Cardoso et al., 2017; Reis et al., 2020).

A cárie possui alta incidência em crianças, e está entre as principais causas da perda precoce de dentes decíduos (Laranjo et al., 2017). É uma doença multifatorial, decorrente de alimentação cariogênica, levando a desmineralização do esmalte dentário caso não ocorra o controle da doença através da correta higienização dos dentes e remoção de biofilme (Dias et al., 2018; Alves \& Severi, 2016).

Para Cardoso et al. (2017), pacientes infantis devem fazer consultas regulares à odontopediatra aliada à orientação de higiene bucal para a prevenção da cárie dentária e, consequentemente, evitar tratamentos invasivos no futuro. Entretanto, quando já houve a perda do elemento dental, os mantenedores de espaço estão indicados (Rodrigues et al., 2019).

De acordo com Ota et al., (2014), Pereira e Miasato (2010), Dainezi et al., (2015), o recurso terapêutico através da reabilitação estética-funcional é de grande valia após a perda prematura de dentes decíduos, pois ajudará o paciente no resultado estético visando a elevação da autoestima do mesmo, bem como melhora significativa na fonação e deglutição. Também evitará a extrusão do dente antagonista. Em contrapartida, Vinothini et al. (2019) sugerem que não é possível ter uma visibilidade adequada do sucessor, e que existem riscos de irritação da mucosa e possibilidade de contato prematuro.

O mantenedor de espaço do tipo banda-alça deve ser usado quando há perda precoce de primeiro ou segundo molar decíduo, em apenas um hemiarco (Lira et al., 2019; Janson et al., 2013). Após a perda do primeiro molar decíduo, o mantenedor deverá permanecer instalado até a erupção do sucessor permanente (Sousa et al., 2010).

Segundo Lira et al. (2019), além de conservar o espaço até a irrupção dos pré-molares na cavidade bucal, este dispositivo evita mesialização do dente adjacente, mal oclusões futuras e não necessita da colaboração do paciente. Com base em um estudo feito por Ahmad et al., (2018), verificou-se perda de cimento usado para fixar a banda ortodôntica no dente pilar, e lesões de tecidos moles após a descimentação. Além disto, o banda-alça não impede a extrusão do elemento dental antagonista (Almeida, 2013).

Com a evolução dos materiais ao longo do tempo, e avaliando as possíveis desvantagens relacionadas ao banda-alça, criou-se o mantenedor de espaço de resina composta reforçada com fibra de vidro e polietileno. O mesmo se adequa à diferentes anatomias dentárias e contornos de arco, é mais aceito esteticamente, e possui fácil higienização (Garg et al., 2014). 
O mantenedor de espaço com ligação direta é uma alternativa viável ao banda-alça, podendo também prevenir o movimento rotacional dos dentes (Ahmad et al., 2018). Todavia, foi observado por Ramakrishnan et al., (2019), que há risco de descolagem do compósito no esmalte resultando na deglutição por parte do paciente.

Sendo assim, o mantenedor de espaço banda-alça convencional ainda realiza um importante papel na manutenção do espaço fisiológico, quando da perda precoce de dentes e é uma alternativa mais segura tanto ao paciente, quanto aos dentes onde se ancora e apoia. Além disso, não impede que as demais funções fisiológicas destes dentes ocorram corretamente (Lira et al., 2019; Almeida, 2013).

\section{Conclusão}

A reabilitação em perda unilateral do primeiro molar decíduo com o uso do mantenedor de espaço banda-alça apresenta eficácia em seus resultados, impedindo a mesialização do dente adjacente e preservando o espaço para a adequada erupção dos dentes sucessores permanentes. O mantenedor de espaço banda-alça é considerado um dispositivo de fácil confecção e ótimo custo-benefício, sendo uma excelente opção de tratamento para pacientes pediátricos, pois não dependerá da cooperação do mesmo e poderá prevenir problemas de má oclusão.

\section{Referências}

Ahmad, A. J., Parekh, S., \& Ashley, P. F. (2018). Methods of space maintenance for premature loss of a primary molar: a review. European Archives of Paediatric Dentistry, 19(5), 311-320.

Almeida, R. R. (2013). Ortodontia preventiva e interceptora: mito ou realidade? Editora DentalPress, 96.

Alves, K. T., \& Severi, L. S. P. (2016). Componentes salivares associados à prevenção da cárie dental - revisão de literatura. Revista de Odontologia da Universidade Cidade de São Paulo, 28(1), 244-262.

Amorim, C., et al. (2019). Direct bonded space maintainer: an alternative in clinical practice after unilateral premature loss of posterior primary tooth. Revista Científica do CRO-RJ (Rio de Janeiro Dental Journal, 4(1).

Cardoso, A. A., et al. (2017). Abordagem integral em odontopediatria: relato de caso clínico. Rev. Assoc. Paul. Cir. Dent., 71(1), 25-29.

Dainezi, V. B., et al. (2015). Reabilitação estética e funcional na primeira infância: relato de caso. Rev. Assoc. Paul. Cir. Dent., 69(4), $387-393$.

De Araújo, L. F., et al. (2018). Cárie precoce de infância: uma visão atual em odontopediatria. Revista Uningá, [S.I],55(S3), 106-114.

Dias, G. F., et al. (2018). Reabilitação estética e funcional em paciente com cárie precoce da infância: relato de caso. Revista de Odontologia da Universidade Cidade São Paulo, 30(3), 314-322.

Dos Reis, J. S., et al. (2018). Traumatismo em dente decíduo, sequela e manutenção de espaço. Revista Uningá, [S.I], 55(S3), 20-28.

Echevarría-Medieta, S., et al. (2019). Mantenedor de Espacio Estético-Funcional em Odontopediatría: reporte de caso. KIRU, 16(2), 81-91.

Garg, A., et al. (2014). "Metal to resin": A comparative evaluation of conventional band and loop space maintainer with the fiber reinforced composite resin space maintainer in children. J Indian Soc Pedod Prev Dent, 32(2), 111-116.

Guimarães, C. A., \& Oliveira, R. C. G. (2017). Perda precoce de dentes decíduos relato de caso clínico. Revista Uningá, Maringá, 29(2), 28-33

Janson, G., et al. (2013). Introdução a Ortodontia, Editora Artes Médicas Ltdas., 108-109.

Laranjo, E., et al. (2017). A cárie precoce da infância: uma atualização. Revista Portuguesa de Medicina Geral e Familiar, 33(6), 426-429.

Lira, A. L. S., et al. (2019). Deciduous tooth early loss prevalence in posterior region and indication of band-loop space maintainer. Brazilian Dental Science, 22(3), 321-327.

Menegaz, A. M., et al. (2015). Efetividade de mantenedores de espaço em odontopediatria: revisão sistemática. RFO, Passo Fundo, $20(2), 252-257$.

Nobrega, M. L., Barbosa, C. C. N., \& Brum, S. C. (2018). Implicações da perda precoce em odontopediatria. RevistaPró-UniverSUS., 09(1), 61-67.

Ota, C. M., et al. (2014). Mantenedor fixo estético-funcional como tratamento para perda precoce de dentes decíduos anteriores. Rev. Assoc. Paul. Cir. Dent., v. 68, n. 4 , p. $308-311,2014$.

Pereira, L., \& Miasato, J. M. (2010). Mantenedor de espaço estético-funcional em odontopediatria. Revista de Odontologia da Universidade Cidade de São Paulo, 22(2), 154-162. 
Research, Society and Development, v. 10, n. 16, e262101624021, 2021

(CC BY 4.0) | ISSN 2525-3409 | DOI: http://dx.doi.org/10.33448/rsd-v10i16.24021

Pawar, B. A. (2019). Maintenance of space by innovative three-dimensional-printed band and loop space maintainer. Journal of Indian Society of Pedodontics and Preventive Dentistry, Mumbai. 37(2), 205-8.

Ramakrishnan, M. et al., (2019). Survival rate of different fixed posterior space maintainers used in Paediatric Dentistry - A systematic review. Saudi Dent J., 31(2), 165-172.

Reis, N. L. S., et al. (2020). Consequências da negligência da saúde bucal em dentes decíduos. Ciências Biológicas e da Saúde Unit, 6(2), 62-72.

Rodrigues, F. V., et al. (2019). Aesthetic and Funcional Rehabilitation in Peaditric Dentstry Patient: Case Report. J. Health Sci., $21(1), 77-81$.

Silva, A. A., et al. (2020). Exodontia do primeiro molar decíduo, seguido de adaptação de mantenedor de espaço tipo banda alça: Relato de caso. Braz. J. Of Develop., Curitiba, 6(10), 80199-80215.

Silva, L. A. M., et al. (2020). Utilização dos mantenedores e recuperadores de espaço na ortodontia interceptativa: Revisão dos conceitos atuais. Research, Society and Development, $9(11), 1-13$.

Sousa, E. S. R., et al. (2010). Manutenção de espaço na dentadura decídua - relato de caso clínico. Brazilian Journal of Health, 1(1), 47-53.

Vinothini, V., et al. (2019). Functional Band and Loop Space Maintainers in Children. Hindawi, CaseReports in Dentistry, 2, 1-4.

Witti, D., et al. (2018). Uso de mantenedor de espaço fixo não funcional em dentição decídua - relato de caso. Ação Odonto, 2. 